\title{
Relação entre parâmetros de monitorização ambulatorial de pressão arterial e o índice ambulatorial de rigidez arterial
}

\author{
Camilo Palencia-Tejedor ${ }^{*}$ \\ Ruth Azuaje-González ${ }^{* *}$ \\ Óscar Barrios-Benedeti ${ }^{* * *}$ \\ José Ayala-Hernández ${ }^{* * * *}$ \\ Carlos Oberto-Machado ${ }^{* * * * *}$ \\ Florángel Salazar-Estéfano ${ }^{* * * * *}$
}

\section{RESUMO}

Objetivo: analisar a relação existente entre os parâmetros de avaliação da monitorização ambulatorial de pressão arterial (pressão arterial, variabilidade, pressão de pulso, carga e padrão) e o índice ambulatorial de rigidez arterial, de um hospital de quarto nível na cidade de Caracas, Venezuela. Materiais e Métodos: realizou-se um estudo observacional retrospectivo, com uma amostra significativa de 179 pacientes registrados na base dados de monitorização ambulatorial de pressão arterial, na consulta cardiometabólica de um hospital, no período compreendido entre junho a dezembro de 2014. Resultados: analisaram-se as monitorizações ambulatoriais de pressão arterial de 179 pacientes em 24 horas, com idade média de $55 \pm 16$ anos, dos quais o 36,3\% era de sexo masculino e o 87,0\% feminino, com um índice ambulatorial de rigidez arterial médio de $0,43 \pm \mathbf{0}, 17$ e se utilizou a correlação de Pearson, com a pressão de pulso $\mathrm{r}=0,50 p<0,001$, com a idade $\mathrm{r}=$ $0,42 p<0,001$, a pressão arterial sistólica $\mathbf{r}=0,24 p=0,001$, a carga $\mathbf{r}=0,20 p=0,007$. Conclusão: encontrou-se correlação positiva entre o índice ambulatorial de rigidez arterial e os parâmetros de avaliação do índice ambulatorial de rigidez arterial, evidenciando que a rigidez ambulatorial arterial é uma medida fácil de obter, que tem boa relação com fatores de risco conhecidos e pode ser contemplada como parâmetro de controle e seguimento cardiovascular.

PALAVRAS-CHAVE: monitorização ambulatorial, pressão arterial, rigidez arterial ${ }^{* * * * * * *}$.

Para citar este artigo/ Para citar este artículo / To reference this article /

Palencia-Tejedor C, Azuaje-González R, Barrios-Benedeti O, Ayala-Hernández J, Oberto-Machado C, Salazar-Estéfano F. Relação entre parâmetros de monitorização ambulatorial de pressão arterial e o índice ambulatorial de rigidez arterial. Rev. cienc. cuidad. 2017; 14(1): 9-22.

ISSN 1794-9831

E-ISSN 2322-7028

Vol. 14 No. 1

Ene - Jun 2017

Cúcuta, Colombia

Recebido:

31 de maio de 2016

Aprovado:

31 de outubro de 2016

* Médico cirurgião.

Especialista em Medicina Interna. Médico Internista Docente. Clínica Medical Duarte. Universidad de Santander - Sede Cúcuta. Cúcuta, Colômbia. Correio electrónico:kmopala@

hotmail.com

** Médico cirurgião

Especialista em Medicina Interna. Médico Adjunto.

Hospital Militar "Dr.

Carlos Arvelo" campus da Universidad Central de Venezuela. Caracas,

Venezuela. Correio electrónico: jrayalah77@. gmail.com

***Médico cirurgião. Especialista em Saúde Ocupacional, Especialista em Medicina Interna.

Clínica Salud Social.

Sincelejo, Colômbia

Correio electrónico:

obarrios3@hotmail.com ****Médico cirurgião. Especialista em Medicina Interna. Médico Adjunto. Hospital Militar "Dr.

Carlos Arvelo" campus da Universidad Central de Venezuela. Caracas, Venezuela. Correio

electrónico: jrayalah77@ gmail.com

*****Médico cirurgião. Especialista em Medicina Interna. Médico

Adjunto. Hospital Militar

"Dr. Carlos Arvelo" campus da Universidad

Central de Venezuela.

San José, Venezuela.

Correio electrónico:

carlos.e.oberto.m@gmail com

******Médico cirurgião. Especialista em Medicina Interna. Médico Adjunto.

Hospital Militar "Dr.

Carlos Arvelo" campus da Universidad Central de Venezuela. Caracas, Venezuela. Correio electrónico: florangels@, yahoo.com

******* Descritores em Ciências da Saúde (DeCS), na página http:// decs.bvs.br/E/homepagee htm da Biblioteca Virtual em Saúde do Projeto BIREME, da Organização Mundial da Saúde (OMS) e da Organização Pan-

Americana da Saúde (OPAS). 
ISSN 1794-9831

E-ISSN 2322-7028

Vol. 14 No. 1

Ene - Jun 2017

Cúcuta, Colombia

\section{Relación entre parámetros del monitoreo ambulatorio de presión arterial y el índice de rigidez arterial ambulatorio}

\section{RESUMEN}

Objetivo: analizar la relación existente entre los parámetros de evaluación del monitoreo ambulatorio de presión arterial (presión arterial, variabilidad, presión de pulso, carga y patrón) y el índice de rigidez arterial ambulatorio, de un hospital de cuarto nivel en la ciudad de Caracas, Venezuela. Materiales y Métodos: se realizó un estudio observacional retrospectivo, con una muestra significativa de 179 pacientes registrados en la base datos del monitoreo ambulatorio de presión arterial, en la consulta cardio-metabólica de un hospital, en el periodo comprendido entre junio a diciembre de 2014 . Resultados: se analizaron los monitoreos ambulatorios de presión arterial de 179 pacientes en 24 horas, con edad promedio $55 \pm 16$ años, de los cuales el 36,3\% era de sexo masculino y el 87,0 $\%$ femenino, con un índice de rigidez arterial ambulatorio promedio de $0,43 \pm 0,17$ y se utilizó la correlación de Pearson, con la presión de pulso $\mathrm{r}=0,50 p<0,001$, con la edad $\mathrm{r}=0,42 p<0,001$, la presión arterial sistólica $\mathrm{r}=0,24 p=0,001$, la carga $\mathrm{r}=0,20 p=0,007$. Conclusión: se encontró correlación positiva entre el índice de rigidez arterial ambulatorio y los parámetros de evaluación del índice de rigidez arterial ambulatorio, evidenciando que la rigidez arterial ambulatoria es una medida fácil de obtener, que tiene buena relación con factores de riesgo conocidos y puede ser contemplada como parámetro de control y seguimiento cardiovascular.

PALABRAS CLAVE: monitoreo ambulatorio, presión arterial, rigidez arterial. 


\section{Relationship between parameters of ambulatory blood pressure monitoring and ambulatory arterial stiffness index}

\section{ABSTRACT}

Objective: To analyze the existent relationship between the evaluation parameters of ambulatory blood pressure monitoring (blood pressure, variability, pulse pressure, load and pattern) and the ambulatory arterial stiffness index, from a level IV hospital in the city ofCaracas, Venezuela. Materials and Methods: a retrospective observational study was performed, with a significant sample of 179 patients registered in the database of blood pressure monitoring, in the cardio metabolic consultation unit of a hospital, in the period between June and December of 2014. Results: the blood pressure monitoring of 179 patients were analyzed in 24 hours, with an average age of $55 \pm 16$ years, from which $36.3 \%$ were male and $87,0 \%$ female, with an average of arterial stiffness index of $0,43 \pm 0,17$ and the Pearson's correlations was used, with pulse pressure $\mathrm{r}=0,50 p<0,001$, with the age $\mathrm{r}=0,42$ $p<0,001$, the systolic blood pressure $\mathrm{r}=0,24 p=0,001$, the load $\mathrm{r}=0,20 p=0,007$. Conclusion: a positive correlation was found between the arterial stiffness index and the parameters of evaluation of ambulatory arterial stiffness index, evidencing that the ambulatory arterial stiffness is an easy measure to obtain, that it has a good relation with known risk factors, and can be contemplated as a parameter of control and cardiovascular monitoring.

KEYWORDS: monitoring ambulatory, arterial pressure, vascular stiffness. 
ISSN 1794-9831

E-ISSN 2322-7028

Vol. 14 No. 1

Ene - Jun 2017

Cúcuta, Colombia

\section{INTRODUÇÃO}

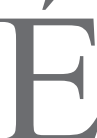
bem conhecido que, quanto maior a idade de uma pessoa, ocorrem mais alterações estruturais no sistema arterial, provocando um aumento da rigidez arterial $^{\dagger}$, a qual se encontra relacionada com a aparição de eventos cardiovasculares maiores (2).

A rigidez arterial está associada com fatores de risco cardiovascular, tais como idade, hipertensão arterial (HTA), tabagismo, nível de colesterol e obesidade (3). Esta rigidez é objeto de pesquisas cada vez mais profundas e atualmente é reconhecida como um índice maior de dano tecidual e como marcador de prognóstico de eventos cardiovasculares (4), o que faz que exista uma relação independente entre a rigidez arterial e a mortalidade (5-9).

As doenças cardiovasculares são responsáveis de quase um terço do total de mortes no mundo (17 milhões ao ano) (10). Dados da Federação Mundial do Coração revelam que as doenças cardiovasculares causam 17,5 milhões de mortes ao ano que em termos comparativos, seria como somar as mortes provocadas por seis das principais causas como são: síndrome da imunodeficiência adquirida (SIDA), tuberculose, malária, diabetes, câncer e patologias respiratórias crónicas. Em outras palavras, por cada pessoa que morre de qualquer tipo de câncer, falecem vinte por doenças cardiovasculares (11).

Sabe-se que a hipertensão arterial (HTA) causa pelo menos o $45 \%$ das mortes por cardiopatias (12). Na Venezuela, a doença cardiovascular é a primeira causa de morte $(21,36 \%$ de mortes ao ano) (13). Na Colômbia, segundo o Observatório Nacional da Saúde, no período 1998-2011 se registraram 628.630 mortes por doença cérebro vascular (ECV) com evidencia de aumento ano após ano desde 1998 com um 21,6\%, até chegar ao ano 2011 com um 25,4\%. Destas mortes, o $56,3 \%$ se relacionaram a doença cardíaca isquêmica, o $30,6 \%$ a doença cerebrovascular e o $12,4 \%$ a doença hipertensiva $(11,14)$.

Em quanto à aparição de eventos cardiovasculares maiores, geralmente considerados como o resultado da exposição ao longo prazo de fatores de risco, como a HTA e a rigidez arterial, entre outras (3). Estes

\footnotetext{
Rigidez arterial: é um termo empregado para definir a capacidade das artérias para expandir-se e contrair-se durante o ciclo cardíaco (1).
}

eventos podem-se explicar pelas diversas mudanças anatômicas e pelo funcionamento do sistema cardiovascular durante o envelhecimento, no qual existe perda de fibras elásticas, calcificação da túnica media y diminuição da produção de óxido nítrico, entre outros, o que explica que, com o endurecimento da parede arterial, se requer mais energia para manter o gasto cardíaco e um maior consumo de oxigênio miocárdico $(11,15-17)$.

Para controlar e medir a pressão arterial se utiliza a monitorização ambulatorial da pressão arterial (MAPA)*, método de importante valor na prática clínica diária, devido a que a medição da pressão arterial é a base para o manejo, seguimento e tratamento da hipertensão arterial, assim como para a avaliação epidemiológica e de pesquisa desta patologia; isto se deve a que a toma de pressão arterial se realiza em todas as situações da vida cotidiana do paciente tais como: no trabalho, no lar e dormindo, entre outras, onde pode-se manifestar a hipertensão arterial e não pode ser avaliada da forma convencional no consultório (19).

Atualmente, existe evidencia clara de que o MAPA é um método que permite não só avaliar o perfil de pressão arterial, senão que tem permitido dilucidar com maior certeza a correlação de morbimortalidade cardiovascular, muito melhor que a toma de pressão arterial de consultório. É por esta razão que os médicos dedicados ao seguimento e tratamento da hipertensão arterial devem estar familiarizados com este método (19).

É através do MAPA como derivado o índice ambulatorial de rigidez arterial ou AASI, por suas siglas em inglês, o qual se obtém da relação dinâmica entre a pressão arterial sistólica (PAS) e pressão arterial diastólica (PAD) durante as 24 horas, calculado pela fórmula um menos a pendente de regressão da pressão arterial diastólica sobre a pressão arterial sistólica, dados obtidos das medições de pressão arterial do MAPA (4).

A fisiopatologia do AASI se explica porque se assume que um aumento dinâmico da pressão provoca um aumento proporcionalmente maior da PAS do que da PAD. Por este motivo, a regressão entre os valores

\footnotetext{
‡ Monitorização ambulatorial de pressão arterial: é um método não invasivo mediante o qual se obtém uma medida da pressão arterial durante 24 horas, fora da consulta médica ou centro de saúde (18).
} 
de PAD e PAS durante o dia tende a uma reta de pendente menor que um. A curva pressão-diâmetro (p-d) arterial é não linear e tem forma exponencial, pelo tanto, um aumento na PAD produz um maior incremento da PAS. Desta maneira, a regressão entre PAD e PAS confirmaria uma pendente menor. Quanto maior seja a rigidez arterial, mais pronunciada é a nãolinearidade $\mathrm{p}-\mathrm{d}$, mais aumenta a PAS em proporção à PAD e menor será a pendente da regressão PAD-PAS e maior o AASI (20).

Dados obtidos do EuroScore mostraram que um valor de AASI menor de 0,45 se correlaciona com risco cardiovascular de $10,4 \%$, entre 0,45 e 0,55 se relaciona com risco cardiovascular de $12,2 \%$ e maior de 0,55 se relaciona com risco cardiovascular de 16,9\% (21) e se considera, então, como medição de rigidez arterial que tem correlação com dano a órgãos-alvo, tais como aumento da microalbuminúria, diminuição da depuração de creatinina, acidente vascular cerebral (AVC), anormalidades na espessura da íntimamédia carotídea e hipertrofia ventricular esquerda (4), e possui valor prognóstico independente de morbimortalidade cardiovascular em hipertensos e de acidente cerebrovascular na população geral; não requer equipamentos, nem pessoal especial para sua medição, o qual se tem comparado com o método de ouro que é a velocidade de onda de pulso (VOP), com bom grau de correlação $r=0,51(6,22-27)$.

Tem-se reportado vários relatórios (17-21) onde o índice ambulatorial de rigidez arterial (AASI) previu a mortalidade cardiovascular e que por acidente cerebrovascular, mais além que a monitorização ambulatorial de pressão arterial (MAPA) de 24 horas, logo acima da pressão de pulso (PP) e além disso, parece ser um melhor previsor que o PP em sujeitos não hipertensos. Atualmente, o MAPA se considera um estudo que deve estar presente em todos os pacientes hipertensos para um correto seguimento e controle (28), pois avalia parâmetros como: o pressão arterial sistólica, o pressão arterial diastólica, o pressão arterial média, o variabilidade, além do pulso radial, carga e padrão, com os quais não existe uma relação completamente estabelecida com o AASI.

O AASI se tem convertido num marcador precoce de risco cardiovascular que poderia permitir um atendimento oportuno dos pacientes com doenças cardiovasculares, a fim de ajudar a evitar os altos custos de atendimento em saúde, assim como, a própria incapacidade dos pacientes que a padecem. Neste sentido, o AASI permite conhecer de forma fácil, económica, fiável e não invasiva, o estado de rigidez arterial dos pacientes que padecem estas doenças, que a sua vez possibilitaria a intervenção farmacológica e mudanças no estilo de vida oportunas, que permitam diminuir, a futuro, as estatísticas de morbimortalidade desta doença e também derivar informação na estratificação de riscos $(5-7,20)$.

Neste sentido, afirma-se, que ao existir uma relação entre os parâmetros de avaliação do MAPA e o AASI, se demostraria a relação quantitativa deste último como fator de risco cardiovascular com outros já bem estabelecidos. Isso permitiria, e propor ao AASI como parâmetro de controle e seguimento dos pacientes com hipertensão arterial e, porque não, de doenças cardiovasculares em geral, pelo que o propósito deste estudo destina-se a analisar a relação existente entre os parâmetros de avaliação do MAPA e o índice ambulatorial de rigidez arterial em pacientes com ou sem patologia de base.

A pesquisa se originou pela necessidade de caracterizar este fator de risco cardiovascular que tem sido objeto de estudos cada vez mais aprofundados, com a intenção de brindar informação sobre sua relação com outros fatores de risco amplamente estudados. Foi desta maneira que se permitiu o acesso à base de dados de uma instituição reconhecida de quarto nível na cidade de Caracas, iniciando assim o estudo.

\section{MATERIAIS E MÉTODOS}

Realizou-se um estudo observacional, retrospectivo, de corte transversal, aleatorizado, dos pacientes registrados na base dados do MAPA da consulta cardio-metabólica de um hospital de quarto nível na cidade de Caracas, Venezuela, onde se tomou como população a 353 pacientes com ou sem patologia de base, e através de uma amostragem simples, utilizando números aleatórios proporcionados pelo software SPSS ${ }^{\circledR}$, versão 16.0, se calculou a amostra com a seguinte fórmula:

$$
n=\left(\frac{\sigma Z_{1-\frac{\alpha}{2}}}{d}\right)^{2}
$$

onde a desvio padrão do AASI foi tomado como 0,5, para um poder estatístico com um alfa $<0,05$,
ISSN 1794-9831

E-ISSN 2322-7028

Vol. 14 No. 1

Ene - Jun 2017

Cúcuta, Colombia 
ISSN 1794-9831

E-ISSN 2322-7028

Vol. 14 No. 1

Ene - Jun 2017

Cúcuta, Colombia um erro beta de 0,1 para um poder de 0,9 , com uma porcentagem de perdas do $10 \%$, resultando em 110 pacientes, com idades de 18 a 90 anos; se excluíram 74 pacientes por registros incompletos, 43 pacientes por registros duplicados, 38 pacientes por registros falidos, 19 pacientes por dados incompletos, ficando um total de 179 pacientes com monitorização ambulatorial de pressão arterial de boa qualidade para ser incluídos no estudo, reclutando-se 69 pacientes adicionais dos calculados na metodologia, o que fez a amostra significativa para este estudo.

Os critérios de inclusão foram: pacientes ambulatórios entre 18 e 90 anos com ou sem patologia de base, incluindo pacientes gravidas, registradas na base de dados do MAPA, que tiveram registros de medições de pressão arterial através deste método de boa qualidade, e se excluiu aqueles pacientes com registros médicos incompletos, medidas falidas ou repetidas.

Para realizar a observação dos pacientes, o MAPA foi gravado num dia comum do paciente, com equipamento marca Welch Allyn® ABPM 6100, utilizando o software proporcionado pelo fabricante. Se considerou aceitável o MAPA quando a recolecção de dados foi legível e se representou pelo $\geq 70 \%$ das gravações totais, instalado no braço não dominante do paciente durante 24 horas, a pressão arterial se tomou a cada 20 minutos durante o dia e a cada 30 minutos durante a noite, se ensinou aos pacientes sobre a importância de continuar suas atividades diárias, e a necessidade de manter tranquilidade ao momento de que a braçadeira do equipamento estivera insuflando para medir a pressão arterial; além disso, se instruiu, aos pacientes para escrever com data e hora qualquer evento estressante que pudera modificar os resultados da proba, para dar-lhe a correta interpretação aos resultados.

O período diurno foi definido como o horário compreendido entre 6:00 e 22:00 horas, e o período noturno foi definido entre 22:00 e 6:00 horas, estes dados foram analisados com uma figura de dispersão onde se calculou a pendente de regressão da pressão arterial diastólica (PAD) sobre a pressão arterial sistólica (PAS); o índice ambulatorial de rigidez arterial (AASI) foi calculado como 1-pendiente de regressão da PAD/PAS de 24 horas $(20-21,30)$.

Se tem definido ao descenso noturno da pressão arterial entre 10 a $20 \%$ com respeito à diurna, seja sistólica e/ou diastólica, como padrão de ritmo circadiano normal ou padrão Dipper; quando este é menor ao $10 \%$ se refere a um padrão Non Dipper. Quando o descenso noturno é maior do que $20 \%$ é um padrão Over Dipper. Quando ocorre uma inversão do ritmo, isto é, uma incremento do valor noturno maior al $10 \%$ do valor diurno se define como padrão Riser (19).

A carga se define como a porcentagem acima do valor normal de pressão arterial (menor 135/85 mmHg e $120 / 70 \mathrm{mmHg}$ diurno e noturno, respectivamente) sendo patológica quando esta por cima de $40 \%$. A pressão de pulso (pulso radial) se definiu como a diferença entre a pressão arterial sistólica e diastólica, associada como fator de risco cardiovascular quando seu valor está acima de $53 \mathrm{mmHg}$ (19). A variabilidade de pressão arterial se definiu como a média de desvio padrão sistólica diurna, a qual se tomou como patológica quando foi maior de $15 \mathrm{mmHg}$ (19). Os pacientes foram divididos em dois grupos, a fim de analisar, de acordo com seu histórico de hipertensão, se estava presente ou não, e depois se contrastaram esses grupos.

Para a análise dos resultados se tabularam os dados utilizando Microsoft ${ }^{\circledR}$ Excel ${ }^{\mathrm{TM}}$ 2013, e posteriormente, eles também foram analisados com o software estatístico SPSS ${ }^{2}$, versão 16.0, Medcalc Stadistical versão 15.6.1. Realizou-se o teste de normalidade baseada em Kolmogorov - Smirnov com os dados que foram maiores de 50 indivíduos se foi menor se utilizou o teste de Shapiro-Wilk para a apresentação dos dados e sua posterior análise.

As variáveis continuas se apresentados como média \pm desvio padrão, e as qualitativas como distribuições de frequência em porcentagens. Utilizou-se o coeficiente de correlação de Pearson $(r)$ e o teste t pareado para grupos independentes para a análise de diferenças entre as variáveis quantitativas obtidas a partir das gravações do MAPA de todos os pacientes, com um intervalo de confiança do $95 \%$, um alfa de 0,05 ; um erro beta de 0,1 ; para um poder de 0,9 numa análise de duas caudas.

O protocolo foi aprovado pelo Comité Académico e de Ética do hospital de quarto nível onde se realizou o estudo, adscrito à Universidad Central de Venezuela, o qual está ligado à Declaração de Helsinki (31) e às apostilas de boas práticas médicas venezuelanas (32), entidade que aprovou a realização do estudo, depois da 
avaliação do protocolo de trabalho apresentado, onde se teve em conta critérios como confidencialidade, desenho da pesquisa, testes a utilizar e o princípio de não maleficência sobre os pacientes, ademais da aceitação e assinatura do consentimento informado de participação no estudo.

\section{OBJETIVOS}

\section{Objetivo geral}

Analisar a relação existente entre os parâmetros de avaliação do MAPA e o índice ambulatorial de rigidez arterial de um hospital de quarto nível na cidade de Caracas, Venezuela.

\section{Objetivos específicos}

- Determinar as características clínicas e demográficas da população de estudo.

- Analisar a correlação entre o AASI e parâmetros do MAPA analisados em subgrupos de hipertensos e não hipertensos.

- Comparar os parâmetros de avaliação do MAPA, AASI e idade entre pacientes hipertensos e não hipertensos.

- Indagar ao AASI como parâmetro de seguimento e controle de pacientes com hipertensão arterial.

\section{RESULTADOS}

O estudo se realizou no período entre o 1 de junho de 2014 até o 1 janeiro de 2015. As limitações encontradas em sua realização foram as relacionadas com a totalidade da informação na base de dados já que cerca do $50 \%$ dos dados a informação obtida foi inicialmente incompleta, duplicados ou falidos, uma situação que reduziu significativamente o número de pacientes com dados de boa qualidade.

\section{Características clínicas e demográficas}

Na tabela 1 pode-se observar a caracterização dos pacientes do estudo, 65 homens $(36,3 \%)$ e 114 mulheres $(87,0 \%)$, com idade média de $55 \pm 16,0$ anos, PAS $122 \pm 12,5$ PAD 71,8 \pm 9,0; pressão arterial média (PAM), 89,7 \pm 9,7; pressão de pulso 50,6 \pm 9,0; carga $23,4 \pm 23,8$. Classificou-se também por antecedentes HTA 131 (73,1\%), não hipertensos 48
(26,8\%), diabéticos 31(17,3\%), não diabéticos 148 $(82,6 \%)$, fumadores $30(16,7 \%)$, não fumadores $149(83,2 \%)$. O AASI se classificou em quartis e 98 pacientes tinham $<0,45(0,30 \pm 0,11), 37$ pacientes $0,45-0,55(0,49 \pm 0,02)$ e 44 pacientes com $0,65 \pm$ 0,09 .

Pode-se apreciar uma clara diferencia entre a porcentagem de homens e mulheres, a favor das mulheres, situação que poderia explicar-se devido a que as mulheres tendem a acudir commais regularidade aos controles médicos do que os homens. Encontrouse também um padrão circadiano de pressão arterial predominantemente patológico, relacionado em porcentagem com pacientes hipertensos, e com a porcentagem de pacientes com AASI maior de 0,45. Como dado adicional, é interessante ver como a história de tabagismo e de diabetes mellitus, fatores claramente associados com risco cardiovascular, tinham uma porcentagem baixa no grupo de estudo.
ISSN 1794-9831

E-ISSN 2322-7028

Vol. 14 No. 1

Ene - Jun 2017

Cúcuta, Colombia 
ISSN 1794-9831

E-ISSN 2322-7028 Vol. 14 No. 1

Ene - Jun 2017

Cúcuta, Colombia

Tabela 1. Características clínicas da população de estudo.

\begin{tabular}{|c|c|}
\hline Características & Total \\
\hline$n$ & 179 \\
\hline Idade & $55,0 \pm 16,0$ \\
\hline Masculino (\%) & $65(36,3)$ \\
\hline Feminino $(\%)$ & $114(87.0)$ \\
\hline \multicolumn{2}{|l|}{ Padrão } \\
\hline Dipper (\%) & $53(29,7)$ \\
\hline Non Dipper (\%) & $85(47,4)$ \\
\hline Riser (\%) & $41(22,9)$ \\
\hline Pressão Arterial Sistólica de 24 horas, $\mathrm{mmHg}$ & $122 \pm 12,5$ \\
\hline Pressão Arterial Diastólica de 24 horas, $\mathrm{mmHg}$ & $71,8 \pm 9.0$ \\
\hline Variabilidade de 24 horas, DE & $13.0 \pm 3.2$ \\
\hline Carga de 24 horas, $\%$ & $23,4 \pm 23,8$ \\
\hline Pressão de Pulso de 24 horas, $\mathrm{mmHg}$ & $50,6 \pm 9,0$ \\
\hline Índice Ambulatorial de Rigidez Arterial & $0,43 \pm 0,17$ \\
\hline$<0,45$ (n) & (98) $0,30 \pm 0,11$ \\
\hline $0,45-0,55(n)$ & (37) $0,49 \pm 0,02$ \\
\hline$>0,55(\mathrm{n})$ & (44) $0.65 \pm 0,09$ \\
\hline Antecedentes & $82,9 \pm 18$ \\
\hline \multicolumn{2}{|l|}{ HTA } \\
\hline SIM (\%) & $131(73,1)$ \\
\hline NÃO (\%) & $48(26,8)$ \\
\hline \multicolumn{2}{|l|}{ DM } \\
\hline SIM $(\%)$ & $31(17,3)$ \\
\hline NÃO (\%) & $148(82,6)$ \\
\hline \multicolumn{2}{|l|}{ Tabagismo } \\
\hline $\operatorname{SIM}(\%)$ & $30(16,7)$ \\
\hline NÃO (\%) & $149(83,2)$ \\
\hline
\end{tabular}

Média \pm Desvio Padrão; HTA, Hipertensão Arterial; DM, Diabetes Mellitus.

Fonte: elaboração própria.

Análise de relação entre os parâmetros do MAPA e o índice ambulatorial de rigidez arterial de pacientes com ou sem patologia de base

Ao relacionar os valores do AASI e os parâmetros do MAPA no grupo completo de estudo, se encontrou que havia uma correlação positiva com a pressão de pulso $r=0,50(p<0,001)$, com a carga $r=0,20(\mathrm{p}=0,007)$, com a pressão arterial sistólica $r=0,24(p=0,001) \mathrm{e}$ com a idade $r=0,42(p<0,001)$, correlação nestes parâmetros são fortemente relacionados como fatores de risco cardiovascular, demostrada neste grupo de estudo, os resultados mostram que a relação que tem sido constante ao longo das pesquisas anteriores, e uma situação fisiológica claramente dilucidada, que corresponde as mudanças anatómicas e funcionais que acontecer com o aumento da idade, como é o fenómeno cativo de rigidez, claramente relacionado de forma linear nestes resultados, onde a correlação entre idade e rigidez arterial, medido através do AASI foi claramente positivo.

Por outra parte, não se encontrou correlação linear com as outras variáveis como a pressão arterial diastólica, 
pressão arterial média e a variabilidade, situação que poderia dever-se a que tal relação se explica mediante outros testes de correlação provavelmente não lineares os quais não se analisaram neste trabalho, como se pode observar na tabela 2 .
ISSN 1794-9831

E-ISSN 2322-7028

Vol. 14 No. 1

Ene - Jun 2017

Cúcuta, Colombia

Tabela 2. Correlação entre o AASI e parâmetros do MAPA em todo o grupo de estudo.

\begin{tabular}{lcc}
\hline Variável & $\boldsymbol{r}$ & $\boldsymbol{p}$ \\
\hline PP & 0,50 & $<0,001$ \\
Carga & 0,20 & 0,007 \\
\hline PAS & 0,24 & 0,001 \\
PAD & $-0,13$ & NS \\
PAM & 0,0001 & NS \\
Variabilidade & $-0,02$ & NS \\
\hline Idade & 0,42 & $<0,001$ \\
\hline
\end{tabular}

PP, pressão de Pulso; PAS, pressão arterial sistólica; PAD, pressão arterial diastólica, PAM, pressão arterial média; NS, não significativa $(\mathrm{p}>0,05)$.

Fonte: elaboração própria.

\section{Resultados da correlação entre o AASI e parâmetros do MAPA analisados em subgrupos de hipertensos e não hipertensos}

Classificou-se os pacientes em hipertensos e não hipertensos, para analisar, por separado a correlação que existia entre o AASI e os parâmetros de avaliação do MAPA em cada grupo de pacientes e se encontrou que no grupo de pacientes hipertensos $\mathrm{n}=131$ existia correlação linear com a PP $(r=0,49 p<0,0001)$, com a carga $(r=0,49 p<0,0001)$, com a PAS $(r=$ $0,23 p<0,0009)$, com a PAD $(r=-0,20 p<0,02)$, e com a idade $(r=0,40 p<0,0001)$; não se encontrou correlação linear com a PAM $(r=-0,04 p=\mathrm{NS})$, como tampouco com a Variabilidade $(r=0,004 p=$ NS).

Estes resultados mostraram como neste grupo de pacientes a relação que existe entre os parâmetros de avaliação do MAPA alterados, é proporcional a grau de rigidez, situação bem explicada desde o ponto de vista fisiopatológico e evidenciada desde o ponto de vista numérico e estatístico.

Assim mesmo, se observou uma clara tendência de correlação invertida entre a variabilidade, a PAM e a rigidez arterial, fenómeno que não se analisou neste trabalho devido a que os testes estatísticos utilizados nesta pesquisa não mediram de forma adequada este tipo de correlação invertida.

Ao analisar o grupo de pacientes não hipertensos se encontrou correlação linear com a idade $(r=0,35$ $p<0,01)$, não se encontrou correlação linear com a PP, carga, PAS, PAD, PAM, variabilidade ( $p=$ NS), o cual mostra uma clara tendência a que os pacientes hipertensos apresentem parâmetros alterados e uma boa correlação com a rigidez arterial medida pelo método ambulatório, como se pode apreciar na tabela 3.

É muito impressionante notar que, em pacientes não hipertensos a rigidez arterial não parecia ter nenhuma relação com os parâmetros do MAPA, achado que poderia explicar-se por que o aumento na rigidez arterial se relaciona com a aparição de HTA e com a alteração dos demais parâmetros de avaliação do MAPA, cujo valor não está alterado nos pacientes não hipertensos.

Com respeito à idade, se encontrou que a correlação com a rigidez arterial é independente do antecedente de HTA, assim que explicado pelas alterações anatómicas e fisiológicos do sistema cardiovascular, devidas ao envelhecimento. 
Tabela 3. Correlação entre o AASI e parâmetros do MAPA em pacientes hipertensos e não hipertensos.

\begin{tabular}{|c|c|c|c|c|c|c|c|}
\hline \multicolumn{4}{|c|}{$\begin{array}{l}\text { Hipertensos } \\
\quad(\mathrm{n}=131)\end{array}$} & \multicolumn{4}{|c|}{$\begin{array}{l}\text { Não hipertensos } \\
\quad(\mathrm{n}=\mathbf{4 8 )}\end{array}$} \\
\hline Variável & $r$ & IC $95 \%$ & $p$ & Variável & $r$ & IC $95 \%$ & $p$ \\
\hline PP & 0,49 & 0,$35 ; 0,61$ & $\mathrm{P}<0,0001$ & PP & 0,14 & $-0,15 ; 0,41$ & NS \\
\hline Carga & 0,22 & 0,$05 ; 0,38$ & $\mathrm{P}=0,0096$ & Carga & $-0,006$ & $-0,29 ; 0,28$ & NS \\
\hline PAS & 0,23 & 0,$06 ; 0,38$ & $\mathrm{P}=0,0092$ & PAS & 0,18 & $-0,11 ; 0,44$ & NS \\
\hline PAD & $-0,20$ & $-0,36 ;-0,03$ & $\mathrm{P}=0,0236$ & PAD & 0,14 & $-0,15 ; 0,40$ & NS \\
\hline PAM & $-0,04$ & $-0,21 ; 0,12$ & NS & PAM & 0,14 & $-0,15 ; 0,41$ & NS \\
\hline Variabilidade & $-0,004$ & $-0,17 ; 0,16$ & NS & Variabilidade & $-0,21$ & $-0,47 ; 0,07$ & NS \\
\hline Idade & 0,40 & 0,$24 ; 0,54$ & $\mathrm{P}<0,0001$ & Idade & 0,35 & 0,$07 ; 0,57$ & $\mathrm{P}=0,0156$ \\
\hline
\end{tabular}

PP, pressão de Pulso; PAS, pressão arterial sistólica; PAD, pressão arterial diastólica, PAM, pressão arterial média; NS, não significativa $(\mathrm{p}>0,05)$.

Fonte: elaboração própria.

Resultados da comparação entre os parâmetros do MAPA, AASI e idade entre pacientes hipertensos e não hipertensos

Quando se comparou o subgrupo de pacientes hipertensos com seus parâmetros de avaliação do MAPA se encontrou correlação positiva com a PP $\operatorname{com} r=0,49 \operatorname{com}(\mathrm{IC}=0,35 ; 0,61 \mathrm{P}<0,0001)$, a carga $r=0,22 \quad(\mathrm{IC}=0,05 ; 0,38 \mathrm{P}=0,0096)$, com a PAS $r=$ $0,23(\mathrm{IC}=0,06 ; 0,38 \mathrm{P}=0,0092)$, a PAD $r=-0,20(\mathrm{IC}=$ $-0,36 ;-0,03 \mathrm{P}=0,0236)$ e com a idade $r=0,40(\mathrm{IC}=$
0,24; 0,54 $\mathrm{P}<0,0001$ ), dados que ao contrastava com os pacientes não hipertensos mostra a escassa relação estatística a exceção da idade com $\mathrm{r}=0,35$ ( $\mathrm{IC}=0,07$; $0,57 \mathrm{P}=0,0156$, o que indica uma clara relação com o AASI e a idade, como se pode observar na tabela 4.

A diferença encontrada entre o AASI dos pacientes hipertensos e não hipertensos é um dado quantitativo que destaca a presença desta nos pacientes do grupo de estudo, e que vale a pena ter em conta em quanto a controle e seguimento se trata.

Tabela 4. Comparação dos parâmetros do MAPA, AASI e idade entre pacientes hipertensos e não hipertensos.

\begin{tabular}{lcccc}
\hline Parâmetro & $\begin{array}{c}\text { Hipertensos } \\
\text { Média }\end{array}$ & $\begin{array}{c}\text { Não hipertensos } \\
\text { Média }\end{array}$ & IC 95\% & $\begin{array}{c}\text { Significância } \\
\text { Estatística }\end{array}$ \\
\hline AASI & 0,45 & 0,36 & $-0,15 ;-0,03$ & $p=0,0012$ \\
\hline PP & 52 & 47,01 & $-7,94 ;-2,08$ & $p=0,0009$ \\
Carga & 25,4 & 18,0 & $-15,29 ; 0,49$ & NS \\
Variabilidade & 13,2 & 12,3 & $-2,00: 0,16$ & NS \\
\hline Idade & 57,2 & 48,4 & $-14,00 ;-3,60$ & $p=0,0010$ \\
\hline PAS & 123,3 & 120,1 & $-7,37 ; 0,95$ & NS \\
PAM & 89,6 & 89,9 & $-2,99 ; 3,53$ & NS \\
PAD & 71,3 & 73,1 & $-1,25 ; 4,75$ & NS \\
\hline
\end{tabular}

PP, pressão de Pulso; PAS, pressão arterial sistólica; PAM, pressão arterial média; PAD, pressão arterial diastólica. NS, não significativa ( $\mathrm{p}>0,05)$

Fonte: elaboração própria. 
Na figura 1 se observa a correlação entre o AASI dos pacientes hipertensos e não hipertensos no grupo de estudo, que se apresenta mediante uma figura e analisado através do teste t pareado, onde se encontrou diferença estatisticamente significativa entre as variáveis analisadas $\mathrm{p}<0,0012$, evidenciando graficamente a diferença entre ambos subgrupos de estudo.
ISSN 1794-9831

E-ISSN 2322-7028

Vol. 14 No. 1

Ene - Jun 2017

Cúcuta, Colombia

Figura 1. Comparação entre o AASI dos pacientes hipertensos e não hipertensos

$$
P=0,0012
$$

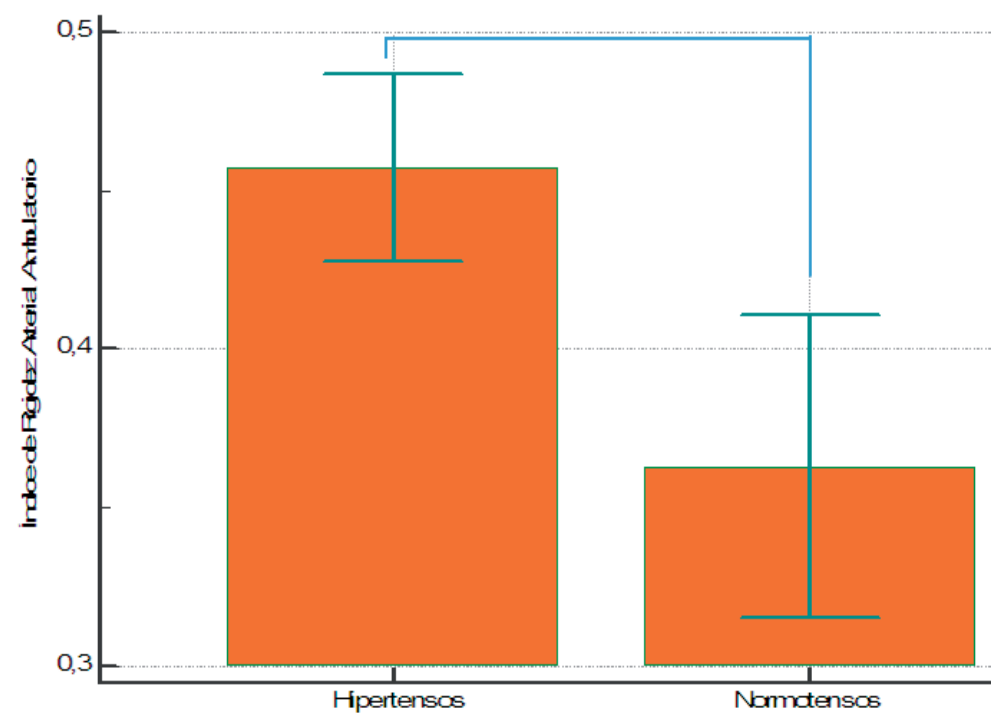

Fonte: elaboração própria.

Em quanto se contrastaram as variáveis de estudo dos pacientes hipertensos e não hipertensos, se encontrou diferença significativa entre os valores de AASI $(\mathrm{IC}=-, 15 ;-0,03 \mathrm{P}=0,0012), \mathrm{PP}=(\mathrm{IC}=-7,94 ;-2,08$ $\mathrm{P}=0,0009)$, idade $(\mathrm{IC}=-14,00 ;-3,60 \mathrm{P}=0,0010)$, não se encontrando diferença estatística quando se compararam os demais parâmetros de avaliação, tais como a variabilidade, PAS, PAD, PAM e carga.

\section{DISCUSSÃO}

Desde o estudo de Li et al. (25) quem encontraram correlação entre o AASI e a VOP $r=0,51$, se tem produzido uma série de trabalhos onde se tem relacionado este índice com marcadores de lesão de órgãos-alvo (4), e também como previsor de eventos cardiovasculares maiores e mortalidade tanto em pacientes hipertensos como em não hipertensos, e hoje se sugere que o AASI pode ser útil para estratificação do risco cardiovascular (5-8). O MAPA é uma ferramenta útil que se tem utilizado, através dos anos, para diagnóstico e seguimento do paciente hipertenso, onde se avaliam parâmetros como PP; carga, variabilidade, PAS, PAD, PAM, frequência cardíaca e padrão (19).

Neste estudo se correlacionou positivamente o índice ambulatorial de rigidez arterial (AASI) com a PP ( $r=$ $0,50 p=<0,001)$, carga $(r=0,20 p=0,007)$, idade $(r$ $=0,42 p=<0,001)$, PAS $(r=0,24 p=0,001)$, pudendo sugerir que estas variáveis, também reconhecidas como fatores de risco cardiovascular, poderiam estar relacionadas entre si.

Os resultados, ademais, mostraram uma relação proporcional com a PP $(r=0,50 p=<0,001)$, iguais aos obtidos no estudo de Simonetti et al. (33), onde se demostrou que o AASI estava aumentado tanto em crianças quanto em adultos hipertensos e a correlação com a PPrespectivamente foi $(r 2=0,1428, p=0,0001)$ $(r 2=0.4603, p=0,0001)$. Resultados similares se observaram no estudo de Dolan et al. (5), onde se encontrou uma tendência à relação proporcional entre pressão de pulso, idade e AASI.

Assim mesmo, no estudo de Lee et al. (34) se encontrou uma correlação positiva entre o AASI e a PP $(r=0,36$ 
ISSN 1794-9831

E-ISSN 2322-7028

Vol. 14 No. 1

Ene - Jun 2017

Cúcuta, Colombia $\mathrm{p}=<0,001)$, com a PAS $(\mathrm{r}=0,23 \mathrm{p}=<0,001)$ e com a idade $(r=0,18 p=<0,001)$, dados que revelam a correlação existente entre os parâmetros do MAPA em paciente hipertenso.

No estudo, a análise por subgrupos mostrou que a correlação entre o AASI e os parâmetros do MAPA foi praticamente nulo nos pacientes não hipertensos, com exceção da idade, que mostrou correlação positiva em ambos os grupos $(r=0,40$ y $r=0,35)$ respectivamente, o que poderia explicar-se pelas mudanças do sistema cardiovascular devidas ao envelhecimento (15-17).

Ao comparar as diferenças entre as variáveis do estudo, entre os dois subgrupos, se descobriu que havia diferença significativa entre o AASI $(=$ $0,45$ vs $r=0,36 p=0,0012)$, a PP $((=0,45$ vs $r$ $=0,36 p=0,0012)$ e a idade $(=57,7$ vs $48,4 p=$ 0,001 ), relações que mostram que no subgrupo de pacientes hipertensos havia mais tendência a parâmetros alterados como também a um AASI mais alto, em contraste com o subgrupo de pacientes não hipertensos; e é lógico observar que os pacientes hipertensos tinham tendência a ser maiores e com maior rigidez.

Estes dados aportam informações úteis que poderia servir de referência para próximos estudos, a fim de evidênciar uma relação entre fatores de risco cardiovasculares e o índice ambulatorial de rigidez arterial.

\section{CONCLUSÕES}

Nas características clínicas e demográficas da população se observou uma tendência clara, a favor das mulheres, a acudir com mais regularidade à consulta médica, demostrando com estes resultados que o gênero feminino tem maior tendência ao autocuidado. Encontrou-se também que o padrão patológico esteve associado com os pacientes hipertensos e com os pacientes com AASI elevado; é muito relevante ver como a história de tabagismo e de diabetes mellitus ocupou uma porcentagem muito baixa em sua associação nos pacientes de estudo.

Neste estudo foi possível demonstrar que existe relação linear entre o AASI e alguns parâmetros do MAPA no grupo estudado, tais como são pressão de pulso, carga, pressão arterial sistólica e idade, parâmetros fortemente relacionados com eventos cardiovasculares maiores como infarto agudo do miocárdio, doença cerebrovascular e morte por causa cardiovascular.

Estes resultados têm sido constantes ao longo das pesquisas anteriores, ressaltando a correlação claramente dilucidada entre o AASI e a idade, os quais tem relação com mudanças anatómicas e funcionais que acompanham ao envelhecimento, como é o fenómeno ativo de rigidez, relacionado de forma linear nestes resultados.

Com respeito a outras variáveis do MAPA pressão arterial diastólica, pressão arterial média, e variabilidade-, não se encontrou correlação linear provavelmente porque a relação entre estas variáveis deve ser não linear. Em estudos futuros deve ser analisado com testes de correlação diferentes.

A análise da correlação entre o AASI e parâmetros do MAPA mostrou uma correlação linear importante com a PP, a carga, PAS, PAS e a idade nos pacientes hipertensos, não evidenciando-se esta correlação nos pacientes não hipertensos, com exceção da idade, que teve correlação em ambos grupos, fenômeno pode explicar-se pelas mudanças fisiopatológicas que acompanham o envelhecimento, confirmando desta maneira a relação proporcional que existe entre estas variáveis, que são bem conhecidas como fatores de risco cardiovascular nos pacientes hipertensos no grupo avaliado.

A comparação entre os parâmetros de avaliação do MAPA, AASI e idade em pacientes hipertensos e não hipertensos evidenciou que nos pacientes hipertensos o AASI, a idade e a pressão de pulso tinham uma diferença estatisticamente significativa com respeito aos não hipertensos, o que reafirma a hipótese de que as mudanças patológicas estão usualmente presentes nos pacientes hipertensos; em outras palavras, a rigidez arterial medida pelo método ambulatório nestes resultados mostrou estar aumentada nos pacientes hipertensos, situação que poderia explicarse pela fisiopatologia da hipertensão arterial.

\section{CONFLITO DE INTERESSES}

Os autores declaram não ter nenhum conflito de interesses. 


\section{REFERÊNCIAS BIBLIOGRÁFICAS}

1. Bia $D$, Zócalo Y. Rigidez arterial: evaluación no invasiva en la práctica clínica. Importancia clínica y análisis de las bases metodológicas de los equipos disponibles para su evaluación. Rev.Urug.Cardiol. 2014; 29(1): 39-59.

2. Forcada P, Melgarejo E, Echeverri D. Cuantificación de la rigidez arterial: de lo básico a lo clínico. Rev Colomb Cardiol. 2015; 22: 69-71.

3. Estadella C, Vázquez S, Oliveras A. Rigidez arterial y riesgo cardiovascular. Hipertens riesgo vasc. 2010; 27(5): 203-210.

4. Leoncini G, Ratto E, Viazzi F, Vaccaro V, Parodi A, Falqui V, et al. Increased Ambulatory Arterial Stiffness Index Is Associated With Target Organ Damage in Primary Hypertension. Hypertension. 2006; 48: 397-403.

5. Dolan E, Thijs L, Li Y, Atkins N, McCormack P, McClory S, et al. Ambulatory arterial stiffness index as a predictor of cardiovascular mortality in the Dublin Outcome Study. Hypertension. 2006; 47(3): 365- 370.

6. Kikuya M, Staessen J, Ohkubo T, Thijs L, Metoki H, Asayama K, et al. Ambulatory arterial stiffness index and 24-hour ambulatory pulse pressure as predictors of mortality in Ohasama, Japan. Stroke. 2007; 38(4): 1161-6.

7. Aznaouridis K, Vlachopoulos C, Protogerou A, Stefanadis C. Ambulatory Systolic-Diastolic Pressure Regression Index as a Predictor of Clinical Events A Meta-Analysis of Longitudinal Studies. Stroke. 2012; 43(3): 733-739.

8. Oliver J, Webb D. Noninvasive assessment of arterial stiffness and risk of atherosclerotic events. Arterioscler Thromb Vasc Biol. 2003; 23(4): 554-566.

9. Sánchez R, Ayala M, Baglivo H, Velázquez C, Burlando G, Kohlmann O. Guías Latinoamericanas de Hipertensión Arterial. Rev Chil Cardiol. 2010; 29: 117-144.

10. World Health Organization (WHO). Causes of death 2008: data sources and methods Department of Health Statistics and Informatics World Health Organization. Geneva: OMS; 2011. p. 1-28.

11. Gómez J. Morbimortalidad cardiovascular en el mundo. Rev. Colomb. Cardiol. 2012; 19(6): 298-299.

12. Lim S, Vos T, Flaxman D, Danaei G, Shibuya K, Adair H, et al. A comparative risk assessment of burden of disease and injury attributable to 67 risk factors and risk factor clusters in 21 regions, 1990-2010: a systematic analysis for the Global Burden of Disease Study 2010. The Lancet. 2012; 380 (9859): 2224-60.

13. Ministerio del Poder Popular para la Salud. Anuario de mortalidad. Venezuela: MPPS; 2014. p. 10-11.

14. Observatorio Nacional de Salud. Primer Informe ONS. Aspectos relacionados con la frecuencia de uso de los servicios de salud. Mortalidad y discapacidad en Colombia. 2011. Bogotá D.C.; ONS; 2013.

15. Milan A, Tosello F, Veglio F, Vairo A, Leone D, Chiarlo M, et al. Arterial stiffness: from physiology to clinical implications. High Blood Press Cardiovas Prev. 2011; 18(1):1-12.

16. Mangas A, Santi M, Barba A, Toro R. Arteriosclerosis. Patogenia de la arteriosclerosis. Mecanismos celulares y moleculares implicados en la aterogénesis. Medicine. 2005; 9 (38): 2495-2505.

17. Zieman S, Melenovsky V, Kass D. Mechanisms, pathophysiology, and therapy of arterial stiffness. Arterioscler Thromb Vasc Biol. 2005; 25(5): 932-943.

18. Contreras E, Zuluaga S. Monitoreo ambulatorio de presión arterial. Rev Mex Cardiol. 2010; 21(1): 25-30.

19. López J, Seijas O, Hernández R, Vega A, Zapata J, Gómez J, et al. Norma Venezolana para el Monitoreo Ambulatorio de Presión Arterial. Revista Latinoamericana de Hipertensión. 2007; 2(1): 1-9.

20. Benetos A, Lacolley P. From 24-Hour Blood Pressure Measurements to Arterial Stiffness. [Editorial]. Hypertension. 2006; 47: 327-328.

21. Bendersky M, Baroni M, Cruz M, Dellamora A, Balestrini C, Serra C, et al. Rigidez arterial ambulatoria. Un nuevo método para mejorar la estratificación del riesgo cardiovascular. Rev Fed Arg Cardiol. 2011; 40(2): 158163.

22. Hamilton PK, Lockhart CJ, Quinn CE, Mcveigh GE Arterial stiffness: clinical relevance, Measurement and treatment. Clin Sci. 2007: 113: 157-170.

23. Laurent S, Boutouyrie P, Asmar R, Gautier I, Laloux B, Guize L, et al. Aortic stiffness is an independent predictor of all-cause and cardiovascular mortality in hypertensive patients. Hypertension. 2001; 37(5): 1236-1241.

24. Laurent S, Cockcroft J, Van Bortel L, Boutouyrie P, Giannattasio C, Hayoz D, et al. Expert consensus document on arterial stiffness: methodoelogical issues and clinical applications. Eur Heart J. 2006; 27(21): 2588-2605.

25. Li Y, Wang J, Dolan E, Gao H, Nawrot T, Stanton A, et al. Ambulatory Arterial Stiffness Index Derived From 24-hour Ambulatory. Hypertension 2006; 47(3): 359-364.

26. Hansen TW, Staessen JA, Torp-Pedersen C, Rasmussen S, Li Y, Dolan E, et al. Ambulatory arterial stiffness index predicts stroke in a general population. J Hypertens. 2006; 24(11): 2247-53.

27. Qin T, Jiang H, Jiao Y, Ke Y, Sun N, Wang J. Ambulatory arterial stiffness index correlates with ambulatory pulse pressure but not dipping status in patients with grade 1/grade 2 essential hypertension. 2014; 42(6): 1323-1334. 
ISSN 1794-9831

E-ISSN 2322-7028 Vol. 14 No. 1 Ene - Jun 2017

Cúcuta, Colombia
28. The Task Force for the Management of Arterial Hypertension of the European Society of Hypertension (ESH), European Society of Cardiology (ESC). 2007 Guidelines for the Management of Arterial Hypertension. J Hypertens 2007; 25: 1105-1187.

29. Manterola C, Otzen T. Estudios observacionales. Los diseños utilizados con mayor frecuencia en investigación clínica. Int J Morphol. 2014; 32(2): 634-645.

30. Valero M, Craiem D, Caputo M, Guzmán G, Graf S, Armentano R. Simulación de la influencia de la elasticidad parietal sobre el índice ambulatorio de rigidez arterial AASI. Rev. ing. biomed. 2009; 3(6): 18-25.

31. Christie B. Doctors revise Declaration of Helsinki. BMJ. 2000; (7266): 913.

32. Asamblea de la Federación Médica Venezolana. Código de Deontología Médica. Aprobado durante la LXXVI Reunión Extraordinaria de la Asamblea de la Federación Médica Venezolana. Caracas; 1985.

33. Simonetti G, Von Vigier R, Wühl E, Mohaupt M. Ambulatory arterial stiffness index is increased in hypertensive childhood disease. Pediat Res. 2008; 64(3): 303-7.

34. Lee H, Lim Y, Kim B, Lee K, Lee J, Kim K. The Relationship Between Ambulatory Arterial Stiffness Index and Blood Pressure Variability in Hypertensive Patients. Korean Circ J. 2011; 41(5): 235-240. 